\title{
Measuring Non-uniformities in GaN/AIN Quantum Wells
}

\author{
K. A. Mkhoyan, H. Wu, W. J. Schaff, L. F. Eastman, and J. Silcox, \\ School of Applied and Engineering Physics, Cornell University, Ithaca, NY 14853 \\ School of Electrical and Computer Engineering, Cornell University, Ithaca, NY 14853
}

Recent increased interest in GaN/AlGaN quantum wells (QWs) is primarily governed by possibilities of fabrication new opto-electronic devices such as optical modulators, switchers. Intersubband transitions in these QWs, on which most of devices are based, have subpicosecond recovery times, ${ }^{1}$ which are typically shorter than those of other semiconductors by an order of magnitude. The mobility and distribution of the carriers in these structures are strongly dependent on the roughness of the quantum wells as well as on sharpness of their interfaces. Reliable measurement of the uniformity of these QWs is needed.

For characterization of these GaN/AlGaN QWs, when the width of the wells is as small as 10-15 $\AA$, scanning transmission electron microscopy with $\sim 2 \AA$ probe is ideal for these type of measurements. ${ }^{2}$ The samples of wurtzite GaN/AIN QWs studied here were grown in an MBE system on 2 inch c-plane sapphire wafers. ${ }^{3}$ The structures consist of $20 \mathrm{GaN} / \mathrm{AlN}$ QWs with initially estimated $60 \AA$ AlN barrier layers and 12$14 \AA \mathrm{GaN}$ wells. Several specimens of the GaN/AlN multiple QWs prepared from two different wafers were studied. Cross-sectional specimens were prepared from these wafers by the tripod polishing technique.

To determine the long-range uniformity of the QWs low magnification, composition sensitive, ADF imaging was used. ADF images from two different areas of sample A are presented in Figs. 1. As is seen, while the quantum wells are reproducible throughout the growth process, they exhibit strong waviness along the growth plane with a period of $\sim 50 \mathrm{~nm}$ and a height $\sim 20 \mathrm{~nm}$. Low magnification ADF images of the second sample (sample B) are presented in Fig 2, where two different areas of the same wafer 4-5 mm apart are shown. Note that multiple QWs in this wafer are significantly uniform over the long-range than QWs in sample A. The waviness is almost absent here. Another interesting observation is that the long-range uniformity of the QWs varies from one area to another across the wafer over a few $\mathrm{mm}$.

The other properties of the QWs that need careful considerations are the symmetry of the quantum wells about the center and the sharpness of the interfaces. For these measurements an area of the QWs in sample A with minimum curviness is chosen (see highlighted box in Fig. 1 (b)) and compositional analysis using EELS was carried out. A single quantum well was mapped by measuring the energy loss structure of the structure at $\sim 3 \AA$ intervals with the $\mathrm{Al} \mathrm{L}_{2,3}$-edge, $\mathrm{Ga} \mathrm{L}_{2,3}$-edge and the ADF intensity along the same line (see Fig. 3). As is seen, these QWs are uniform throughout the width despite the long-range waviness of the QWs. The interfaces between the GaN QW and the AlN barrier are almost atomically sharp. To measure the symmetry of the GaN QW across the width in sample B, EELS was again used by probing changes in energy-loss spectra of the Ga $\mathrm{L}_{2,3}$-edge and now N K-edge. Figs. 4 (a)-(e) summarize the results of the measurements. To determine alterations in the spectra of the $\mathrm{N}$ K-edge, each recorded spectrum was fitted to a spectrum composed as a linear combination of two spectra of the $\mathrm{N}$ K-edge in bulk AlN and GaN, $\mathrm{I}_{\mathrm{s}}=$ $\alpha_{1} \mathrm{I}_{\mathrm{AlN}}+\alpha_{2} \mathrm{I}_{\mathrm{GaN}}$. A least squares curve fitting algorithm was applied for this purpose and coefficients $\alpha_{1}$ and $\alpha_{2}$ were obtained for each recorded spectrum. The results for some spectra are presented in Fig. 4 (d). Two sets of data for coefficient $\alpha_{1}$ are presented in 4 (b). These measurements also provide accurate determination of the actual physical width of the wells. They were $10 \AA$ and $12 \AA$ for samples A and B respectively. ${ }^{4}$

\section{$\underline{\text { References }}$}

1. C. Gmachl, H.M. Ng and A.Y. Cho, Appl. Phys. Lett. 79, 1590 (2002).

2. D. A. Muller, at al., Nature 399758 (1999).

3. H. Wu, et al., MRS Symp. Proc. (2002), submitted.

4. The authors thank M. Thomas and Dr. E.J. Kirkland for their technical support. This work is supported by ONR No. N00014-99-10714, NSF No.01243 and DARPA/ARO No. 190210199. 

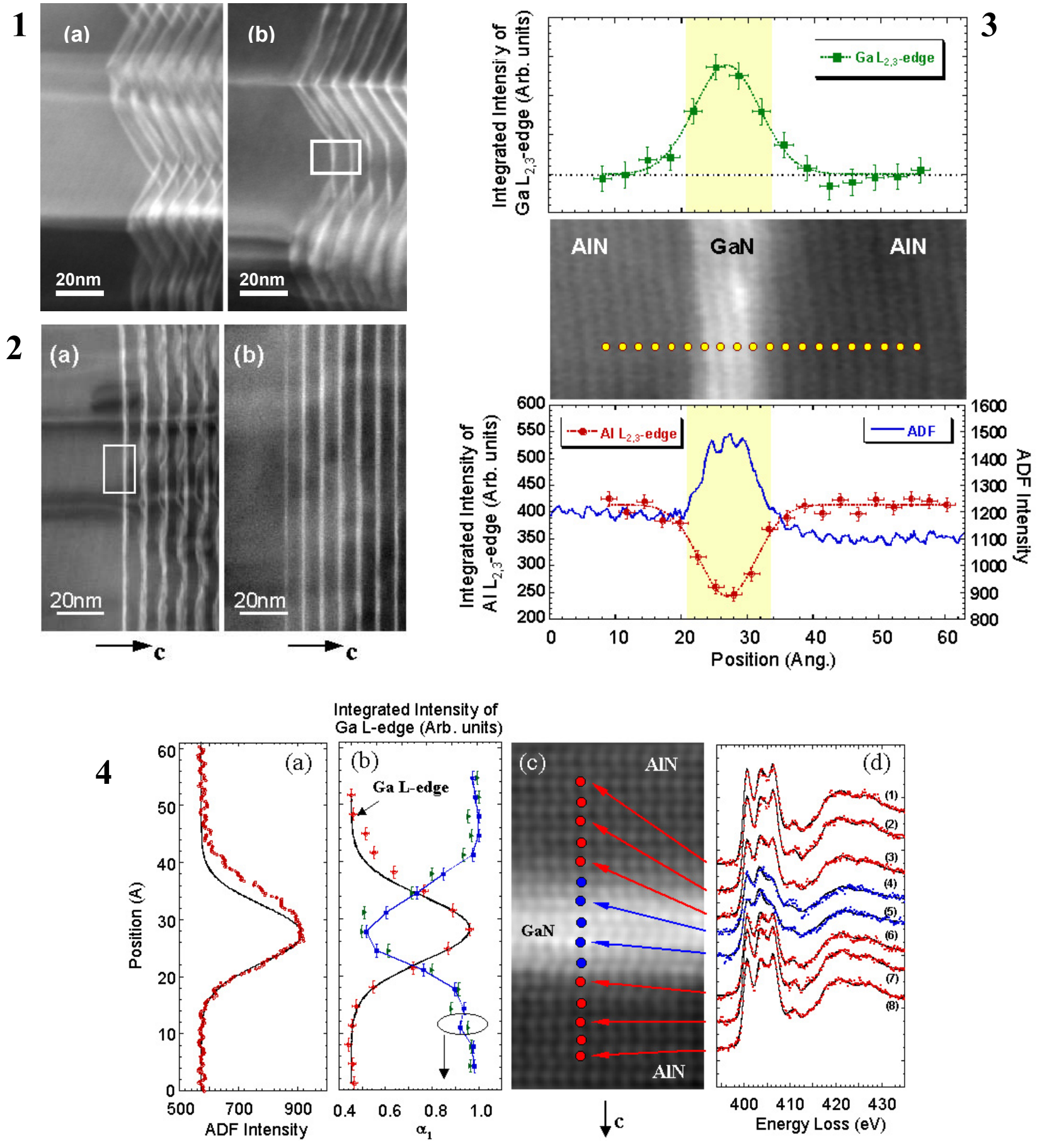

Fig 1. ADF images from two different areas $(a, b)$ of the GaN/AlN multiple quantum wells from sample A.

Fig 2. ADF images of the GaN/AlN multiple quantum wells from sample B. Two different areas 4-5 mm apart are presented (a) and (b). The c-axis of the crystal is also indicated.

Fig 3. High magnification ADF image of the single QW (GaN layer) with mapping path. The integrated intensity of the $\mathrm{Ga}$ and $\mathrm{Al} \mathrm{L}_{2,3}$-edge, and the intensity of the ADF signal across this path are also presented.

Fig 4. The results of probing GaN QW in AlN matrix with $\sim 2 \AA$ focused electron beam: (a) intensity of ADF signal; (b) integrated intensity of $\mathrm{Ga} \mathrm{L}_{2,3}$-edge and coefficient $\alpha_{1}$; (c) high resolution ADF image of the GaN QWwith probing path; (d) spectra of N K-edge recorded across the QW with their best fits. 